\title{
ASYMPTOTIC BEHAVIOR OF SOLUTIONS TO THE STEFAN PROBLEM WITH A KINETIC CONDITION AT THE FREE BOUNDARY
}

\author{
J. N. DEWYNNE ${ }^{1}$, S. D. HOWISON ${ }^{1}$, \\ J. R. OCKENDON ${ }^{1}$ and WEIQING XIE ${ }^{1}$
}

(Received 18 October 1988)

\begin{abstract}
We study the large time behaviour of the free boundary for a one-phase Stefan problem with supercooling and a kinetic condition $u=-\varepsilon|\dot{s}|$ at the free boundary $x=s(t)$. The problem is posed on the semi-infinite strip $[0, \infty)$ with unit Stefan number and bounded initial temperature $\varphi(x) \leq 0$, such that $\varphi \rightarrow-1-\delta$ as $x \rightarrow \infty$, where $\delta$ is constant. Special solutions and the asymptotic behaviour of the free boundary are considered for the cases $\varepsilon \geq 0$ with $\delta$ negative, positive and zero, respectively. We show that, for $\varepsilon>0$, the free boundary is asymptotic to $k \sqrt{t}$, $\delta t / \varepsilon$ if $\delta<0, \delta>0$ respectively, and that when $\delta=0$ the large time behaviour of the free boundary depends more sensitively on the initial temperature. We also give a brief summary of the corresponding results for a radially symmetric spherical crystal with kinetic undercooling and Gibbs-Thomson conditions at the free boundary.
\end{abstract}

\section{Introduction}

We study the qualitative behaviour and special solutions of the Stefan problem with a kinetic condition at the free boundary [5], [6]. Several authors have considered this problem and the existence, uniqueness and regularity of the solution have been obtained (e.g. [14], [15]).

We consider here the one-phase Stefan problem on a semi-infinite strip $[0, \infty)$, with a kinetic condition at the free boundary, unit Stefan number and bounded initial temperature $\varphi(x) \leq 0$, so that the liquid is supercooled.

\footnotetext{
'Mathematical Institute, 24-29 St. Giles, Oxford OX1 3LB, U. K.

(C) Copyright Australian Mathematical Society 1989, Serial-fee code 0334-2700/89
} 
That is, we study the following dimensionless problem which corresponds to the limit of a two phase Stefan problem when the thermal diffusivity in the solid is vanishingly small:

$$
\begin{array}{ll}
u_{t}-u_{x x}=0, & s(t)<x<\infty, t>0 \\
u(x, 0)=\varphi(x) \leq 0, & 0 \leq x<\infty \\
u(s(t), t)=-\varepsilon \dot{s}(t), & t>0 \\
u_{x}(s(t), t)=-\dot{s}(t), & t>0
\end{array}
$$

where $\varepsilon \geq 0$ is a constant, and where $\varphi(x) \in C^{1}[0, \infty)$ is a given function, which is bounded together with its first derivative. The negativity of $\varphi$ ensures that $\dot{s}>0$.

The problem (1.1)-(1.4) reduces to the standard supercooled Stefan problem when $\varepsilon=0$. It is known that when $\varepsilon=0$ the solution of $(1.1)-(1.4)$ can blow up with $\dot{s} \rightarrow \infty$ in finite time for certain initial data $\varphi(x)$, in particular when (but not only when) $\varphi(\infty)<-1[7,9,11]$, and also that the kinetic term with $\varepsilon>0$ prevents blow-up for any initial data $\varphi(x)$, at least for the analogous problem posed on a finite spatial domain (see [15]).

In this paper we give some special solutions and discuss the asymptotic behaviour corresponding to initial data $\varphi(x)$ with

$$
\varphi(x) \rightarrow-1-\delta \text { as } x \rightarrow \infty \text {, }
$$

where $\delta$ is a constant. The plan of the paper is as follows. In Section 2 we review known results on similarity solutions and asymptotic behaviour when $\varepsilon=0$. In Section 3 we present analogous exact solutions for $\varepsilon>0$ and in Section 4, using an integral equation derived from the Laplace transform of (1.1)-(1.4), we analyse the asymptotic behaviour of $s(t)$ as $t \rightarrow \infty$ in the case that the initial data is not compatible with one of the similarity solutions previously noted. In Section 5 we summarise the results of Sections 2-4. Lastly, in Section 6 we give a brief review of the corresponding results for a spherical crystal growing in three dimensions, with surface energy effects incorporated via a Gibbs-Thomson condition on the free boundary. These are qualitatively the same as the one-dimensional results of Sections 2-5. Our analysis here complements the numerical work of Schaefer and Glicksman 
[13]; they pointed out that values of $\delta$ as high as 0.8 are obtainable using certain materials.

\section{Known results and exact solutions when $\varepsilon=0$}

We first review some results for the standard supercooled Stefan problem. Firstly, as a consequence of the finite time blow-up for the initial-value problem with initial data $\varphi(x)$ having $\varphi(x)<-1$ (see $[7,9,11]$ ), we know that there is no solution of (1.1) -(1.4) for large time if $\delta$ is a positive constant. Secondly, when $\delta<0$, there is a similarity solution of the form ${ }^{2}$

$$
u(x, t)=f(x / \sqrt{t}), \quad s(t)=\beta \sqrt{t}
$$

where

$$
f(\xi)=\frac{\beta}{2} \exp \left(\beta^{2} / 4\right) \int_{\xi}^{\beta} \exp \left(-y^{2} / 4\right) d y
$$

and $\beta$ is to be determined from

$$
\beta \exp \left(\beta^{2} / 4\right) \int_{\beta / 2}^{\infty} \exp \left(-y^{2}\right) d y=1+\delta,
$$

which has real positive solutions only if $-1<\delta<0$ [1]. The initial data for this solution is the step function $\varphi(x)=-1-\delta, x \geq 0$.

The asymptotic behaviour

$$
s(t) \sim \beta \sqrt{t}, \quad \text { as } t \rightarrow \infty
$$

was obtained by [3] for any initial data $\varphi(x)$ with $\varphi^{\prime \prime}>0$ and $\varphi(\infty)>-1 / 4$. It is a reasonable conjecture that (2.4) is true for any $\varphi(x)$ with $\varphi(\infty)>-1$ and for which finite-time blow-up does not occur; we shall support this conjecture with asymptotic results in Section 4.

Lastly when $\delta=0$, we can find a travelling wave solution in the form

$$
u(x, t)=\exp (-V(x-V t))-1, \quad s(t)=V t
$$

where $V$ is any positive constant. We remark here that there is no similarity solution of the form (2.1) for $\delta=0$ and no travelling wave solution of the

2 In using $\beta$ here, we are following the notation of Lame \& Clapeyron (1831) [12] who first considered the one-phase Stefan (sic) problem. 
form (2.5) if $\delta<0$. We also note that $V$ in (2.5) is arbitrary, whereas $\beta$ in (2.1) is determined by $\varphi(\infty)$.

\section{Exact solutions for $\varepsilon>0$}

We begin our analysis of the case $\varepsilon>0$ by noting two exact solutions analogous to the solutions given in Section 2 .

(a) $\varepsilon>0, \delta<0$ : similarity solution with $s(t)=\beta \sqrt{t}$

We begin with the case $\delta<0$. We know that there are similarity solutions of the form (2.1) if $\varepsilon=0$. When $\varepsilon>0$, we incorporate the kinetic condition (1.3) by seeking similarity solutions of the form

$$
\begin{gathered}
u(x, t)=f(\xi)+g(\xi) / \sqrt{t}, \quad \xi=x / \sqrt{t}, \\
s(t)=\beta \sqrt{t}, \quad \beta>0 .
\end{gathered}
$$

We find that $f(\xi)$ and $g(\xi)$ satisfy the ordinary differential equations $f^{\prime \prime}+$ $(\xi / 2) f^{\prime}=0, g^{\prime \prime}+(1 / 2)(\xi g)^{\prime}=0$ where primes denote differentiation with respect to $\xi$. Further, from the kinetic and Stefan conditions, (1.3)-(1.4) we find that

$$
f(\beta)=0, \quad f^{\prime}(\beta)=-\beta / 2, \quad g(\beta)=-\varepsilon \beta / 2, \quad g^{\prime}(\beta)=0,
$$

and so

$$
\begin{gathered}
f(\xi)=\frac{\beta}{2} e^{\beta^{2} / 4} \int_{\xi}^{\beta} e^{-y^{2} / 4} d y, \\
g(\xi)=\frac{\beta}{2}\left\{\frac{\beta}{2} e^{-\xi^{2} / 4} \int_{\xi}^{\beta} e^{y^{2} / 4} d y-e^{\left(\beta^{2}-\xi^{2}\right) / 4}\right\} .
\end{gathered}
$$

Since $g(\xi) \rightarrow 0$ as $\xi \rightarrow \infty, \beta$ is determined by (2.3) which, as already noted, has real positive solutions only when $-1<\delta<0$.

We observe that $\beta$ is independent of $\varepsilon$. This surprising result is reminiscent of the fact that, without kinetic undercooling, the corresponding similarity solution for the growth of a spherical solid region expanding into supercooled liquid has a rate of growth unaffected by the inclusion of a Gibbs-Thomson condition at the free boundary [10] (see also Section 6). It is to be contrasted with the results of the next part of this section, where we find a travelling wave solution whose speed does depend on $\varepsilon$.

Finally, we note that $u(x, 0+) \sim O(1 / x)$ as $x \rightarrow 0$, but that finite initial data can be obtained by shifting the origin of $t$. 
(b) $\varepsilon>0, \delta>0$ : travelling-wave solutions

When $\delta>0$, a travelling-wave solution analogous to (2.5) can be found. We seek a solution

$$
\begin{aligned}
u(x, t)=f(z), & z=x-V t \\
s(t)=V t, & V>0 .
\end{aligned}
$$

By direct calculation we establish that

$$
\begin{gathered}
u(x, t)=\exp [-V(x-V t)]-(1+\delta) \\
s(t)=V t=\delta t / \varepsilon .
\end{gathered}
$$

Here the wave speed $V$ is uniquely determined.

We now investigate how the possible travelling-wave solutions of (1.1)(1.4) behave as the parameters $\varepsilon$ and $\delta$ approach zero. Suppose first that $\varepsilon=o(\delta)$ as $\varepsilon \rightarrow 0, \delta \rightarrow 0$. In this case $\varphi(x)$ tends to a step function and the velocity $V$ becomes infinite as $\delta$ and $\varepsilon \rightarrow 0+$, suggesting that there is no solution for the problem with $\varepsilon=0, \delta=0$ when the initial data is a step function. In the case where $\delta=o(\varepsilon)$, we observe that $V \rightarrow 0+$ as $\varepsilon, \delta \downarrow 0$, the corresponding initial function goes to zero and we retrieve the trivial solution, although the limit is not uniform as $x \rightarrow \infty$.

Finally, in the case $\delta=O(\varepsilon)$, we notice that $V=\delta / \varepsilon$ is bounded as $\varepsilon, \delta$ go to zero. This gives the solution (2.5), and underlines the fact that $V$ is indeterminate in the limit $\delta, \varepsilon \downarrow 0$. Note that there is no bounded travelling wave solution of the form (3.5), (3.6) if $\delta$ is negative.

\section{Asymptotic behaviour of $s(t)$ as $t \rightarrow \infty$}

In this section, we discuss the large-time behaviour of the free boundary $s(t)$ by considering an integral equation formulation of problem (1.1)-(1.4). We begin with the assumption that there is indeed a unique classical solution for all $t>0$. This is the case for $\varepsilon>0$, provided the initial data $\varphi(x)$ satisfies some mild conditions, for example $\varphi \in C^{\mathrm{l}}[0, \infty)$ and $\varphi, \varphi^{\prime}$ are bounded (see [14], [15]).

We now investigate the large-time behaviour of solutions with arbitrary bounded smooth initial data satisfying (1.5).

The first step is to reduce (1.1)-(1.4) to an integral equation by applying a Laplace transform in $x$ [8]. We define the transform $\hat{u}(p, t)$ of $u(x, t)$ by

$$
\hat{u}(p, t)=\int_{s(t)}^{\infty} e^{-p x} u(x, t) d x
$$


by a direct calculation using (1.1)-(1.4) we find that

$$
\partial \hat{u} / \partial t-p^{2} \hat{u}=[1+\varepsilon p+\varepsilon \dot{s}] \dot{s} e^{-p s}, \quad \hat{u}(p, 0)=\hat{\varphi}(p)
$$

where $\hat{\varphi}(p)=\int_{0}^{\infty} e^{-p x} \varphi(x) d x$. Thus we have, from (4.2),

$$
\hat{u}(p, t)=e^{p^{2} t}\left[\hat{\varphi}(p)+\int_{0}^{t} \dot{s}(\tau)(1+\varepsilon p+\varepsilon \dot{s}) e^{-p s(\tau)-p^{2} \tau} d \tau\right] .
$$

Since $u(x, t)$ exists and is bounded for all $t$, it follows that $\hat{u}(p, t)$ exists and is bounded for all $t$ and $\operatorname{Re} p>0$. Thus taking $|\arg p|<\pi / 4$ and letting $t \rightarrow \infty$, the quantity in square brackets in (4.3) must vanish identically, yielding

$$
\hat{\varphi}(p)=-\int_{0}^{\infty} \dot{s}(\tau)[1+\varepsilon p+\varepsilon \dot{s}(\tau)] e^{-p s(\tau)-p^{2} \tau} d \tau
$$

Integrating by parts, we obtain another more convenient form of (4.4):

$$
\begin{aligned}
\hat{\varphi}(p)= & -\frac{1}{p}-\varepsilon+p(1+\varepsilon p) \int_{0}^{\infty} e^{-p s-p^{2} t} d t \\
& -\varepsilon \int_{0}^{\infty} \dot{s}^{2} e^{-p s-p^{2} t} d t .
\end{aligned}
$$

The behaviour of $s(t)$ depends on the balance between the terms on the righthand side of (4.5).

In order to obtain the asymptotic behaviour of $s(t)$ as $t \rightarrow \infty$, we must investigate (4.5) as $p \rightarrow 0$, in particular the behaviour of the integrals $\int_{0}^{\infty} e^{-p s-p^{2} t} d t$ and $\int_{0}^{\infty} \dot{s}^{2} e^{-p s-p^{2} t} d t$. We first note that, by a direct calculation, if we take $s(t)=\beta \sqrt{t}$ with $\beta$ a positive constant, then

$$
\begin{aligned}
& p \int_{0}^{\infty} e^{-p s-p^{2} t} d t=\frac{1}{p}\left[1-\beta e^{\beta^{2} / 4} \int_{\beta / 2}^{\infty} e^{-x^{2}} d x\right], \\
& \int_{\eta}^{\infty} \dot{s}^{2} e^{-p s-p^{2} t} d t \sim \log p \sqrt{\eta}, \quad \text { as } p \rightarrow 0,
\end{aligned}
$$

and, if we take $s(t)=V t$ with $V$ a positive constant, then

$$
\begin{gathered}
p \int_{0}^{\infty} e^{-p s-p^{2} t} d t=\frac{1}{p+V} \\
\int_{0}^{\infty} \dot{s}^{2} e^{-p s-p^{2} t} d t=\frac{V^{2}}{(p+V) p} .
\end{gathered}
$$

Comparing these forms for $s(t)$, when the initial data is such that $\hat{\varphi}=$ $-(1+\delta) / p+\hat{\varphi}_{1}$, where $\hat{\varphi}_{1}=o(1 / p)$ as $p \rightarrow 0$ (for example, if $\varphi_{1}(x)$ is bounded and vanishes at $\infty$, or if $\left.\varphi_{1}(x) \sim \sin \omega x\right)$, we conclude that the asymptotic behaviour of $s(t)$ is $\beta \sqrt{t}$ if $\delta<0, \varepsilon \geq 0$, and $V t$ if $\delta>0, \varepsilon>0$; $\beta$ and $V$ are determined as in Sections 2 and 3 respectively. This analysis 
further suggests that in the marginal case $\delta=0, \varepsilon>0$, the free boundary is in general asymptotic neither to $V t$ nor to $\beta \sqrt{t}$ as $t \rightarrow \infty$. We therefore investigate in more detail the remaining case $\delta=0, \varepsilon \geq 0$, where the asymptotic form of $s(t)$ depends more sensitively on $\hat{\varphi}(p)$.

(1a) We start with $\delta=\varepsilon=0$ and choose initial data $\varphi(x)$ with the form $\varphi(x)=-1+\varphi_{1}(x)$ where $\hat{\varphi}_{1}(p)$ is finite (that is, $\varphi_{1}(x)$ is integrable over $[0, \infty))$ and nonzero at $p=0$. Then we find the asymptotic behaviour of the free boundary to be $s(t) \sim V t$ as $t \rightarrow \infty$, with $V=1 / \hat{\varphi}_{1}(0)$. We see from (4.5)-(4.7) that no similar result is valid if $\varepsilon>0$.

(1b) We next discuss the case (still with $\delta=\varepsilon=0$ ) where the initial data satisfies $\varphi(x) \sim-1+c x^{-(1+\gamma)}+o\left(x^{-(1+\gamma)}\right)$ as $x \rightarrow \infty$, so that

$$
\hat{\varphi}(p) \sim-p^{-1}+a p^{\gamma}+o\left(p^{\gamma}\right) \text { as } p \rightarrow 0
$$

where $-1<\gamma<0$ and $a=c \Gamma(-\gamma)$. If, in the first integral of (4.5), we take $s(t) \sim k t^{\alpha}, \frac{1}{2}<\alpha<1$, then, by rescaling time so that $s(t) \sim p t$ (i.e. putting $\left.t=(k / p)^{1 /(1-\alpha)} \tau\right)$ and applying Laplace's method [4] to estimate the behaviour of the integral as $p \rightarrow 0$, we find that

$$
p \int_{0}^{\infty} e^{-p s-p^{2} t} d t \sim \frac{1}{\alpha} \Gamma\left(\frac{1}{\alpha}\right) k^{-1 / \alpha} p^{(1-1 / \alpha)}+o\left(p^{(1-1 / \alpha)}\right)
$$

as $p \rightarrow 0$. This gives an estimate of the order of singularity (with respect to $p$ ) of this integral as $p \rightarrow 0$. The relation between the order $\gamma$ of this singularity and $\alpha$ is depicted in Figure 1.

Comparing (4.9) to (4.8), we see from (4.5) with $\varepsilon=0$ that $s(t) \sim k t^{\alpha}$ where

$$
\alpha=\frac{1}{1-\gamma}, \quad k=\left(\frac{\Gamma(1 / \alpha)}{a \alpha}\right)^{\alpha} .
$$

The inequality $-1<\gamma<0$ implies that $\frac{1}{2}<\alpha<1$. Note that in general $k$ will be real and positive only if $a>0$; that is, there will be a solution only if $\varphi(x) \geq-1$ in the far field. Indeed, it is likely that finite-time blow-up will occur if $a<0$.

This method can clearly be extended to more complicated behaviour of $\hat{\varphi}(p)$.

Note that if we take $\varphi(x)=-1,0 \leq x<\infty$ (i.e. the initial data is a unit step function) then $\hat{\varphi}(p)=-\frac{1}{p}$. Then in (4.5) with $\varepsilon=0$, all the terms cancel except for $p \int_{0}^{\infty} e^{-p s(t)-p^{2} t} d t$, which is strictly positive. Thus the supercooled Stefan problem with unit step function initial data $\varphi(x)$ has no solution that has a Laplace transform (4.1).

(1c) If we consider initial data of the form $\varphi(x)=-1+\varphi_{1}(x)$ where $\hat{\varphi}_{1}(p)$ vanishes at $p=0$, then from parts $(1 \mathrm{a}, \mathrm{b})$ of this section, it is apparent that 


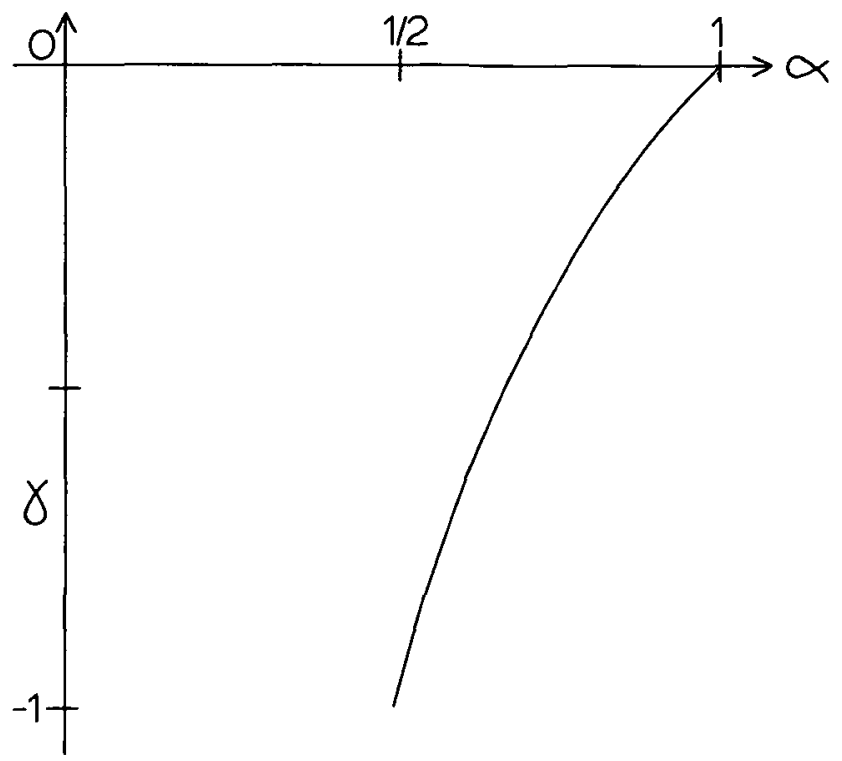

FIGURE 1. Relation between $\gamma$ and $\alpha$ for $\delta=0, \varepsilon=0$.

it is impossible to have $s(t) \sim k t^{\alpha}$ for any $\frac{1}{2} \leq \alpha \leq 1$. We thus look for solutions which have $s(t) \sim k t^{\alpha}$ for $\alpha>1$, as $t \rightarrow \infty$. The estimate (4.9) remains valid for $\alpha>1$ (although a different scaling $\tau=p^{2} t$ is necessary to obtain it). Thus if we assume that $\hat{\varphi}_{1}(p) \sim a p^{\gamma}$ as $p \rightarrow 0$ for $\gamma>0$, we recover (4.10). Clearly, however, this is valid only for $0<\gamma<1$.

The condition that $\hat{\varphi}_{1}(0)=0$ is simply the condition that $\int_{0}^{\infty} \varphi_{1}(x) d x=$ 0 , and since the assumption that $\hat{\varphi}_{1}(p) \sim a p^{\gamma}$ excludes the possibility that $\varphi_{1} \equiv 0$, this implies that $\varphi_{1}(x)$ must change sign. In particular, it implies that there must be regions where $\varphi(x)<-1$.

By analogy with the finite-time blow-up case (where $\dot{s}(t)$ becomes infinite in a finite time) we can regard these cases as infinite-time blow up (since $\hat{s}(t)$ is unbounded at $t \rightarrow \infty$ ). Evidently such infinite time blow up cannot occur if $\varepsilon>0$, for the maximum principle implies that $|\dot{s}| \leq \sup |\varphi(x)| / \varepsilon$ (see [15]).

(1d) Now we consider the case $\varepsilon>0$ and $\hat{\varphi}(p)$ of the form (4.8). Suppose we take $s(t)=k t^{\alpha}, \alpha \in\left(\frac{1}{2}, 1\right)$; then we can obtain an estimate for the second integral in (4.5) in the same manner as for the first integral in (4.5), namely

$$
\varepsilon \int_{0}^{\infty} \dot{s}^{2} e^{-p s-p^{2} t} d t \sim \varepsilon \alpha k^{1 / \alpha} \Gamma\left(2-\frac{1}{\alpha}\right) p^{(1 / \alpha-2)}+o\left(p^{(1 / \alpha-2)}\right) \text { as } p \rightarrow 0 .
$$

The orders of magnitude of these two integrals as $p \rightarrow 0$ (as determined by (4.9) and (4.11)) are shown as functions of $\alpha$ in Figure 2. According to 


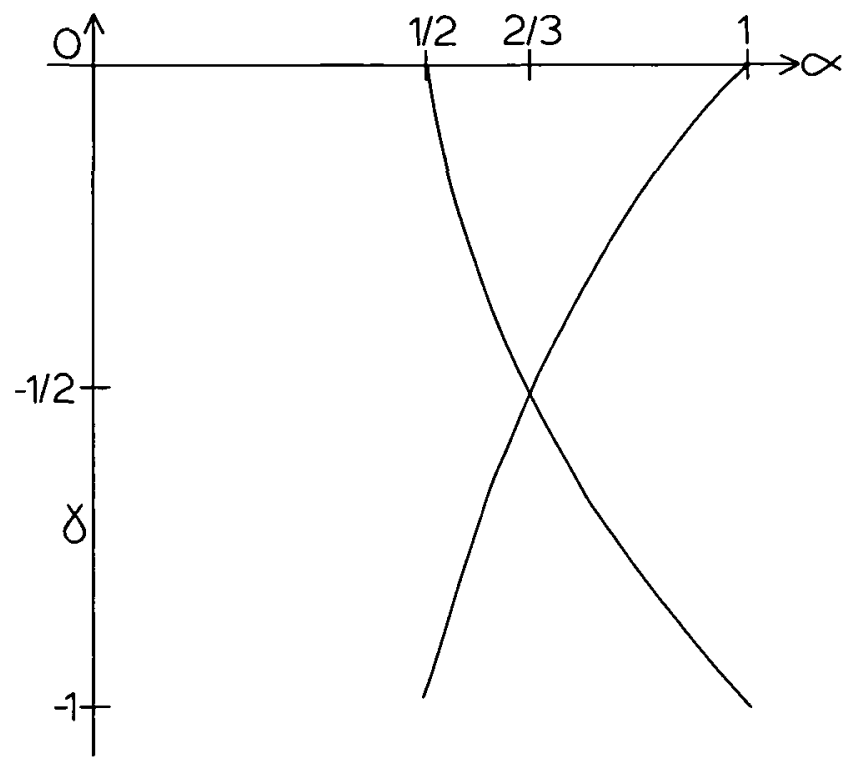

FIGURE 2. Relations between $\gamma$ and $\alpha$ for $\delta=0, \varepsilon>0$.

Figure 2 we see, from (4.5), (4.8)-(4.11), that the following cases must be considered:

(i) $-1<\gamma<-\frac{1}{2}$ : There are two possible choices for $\gamma$ (see Figure 2). The choice we make depends on the sign of $a$ in (4.8). If $a>0$, the first integral in (4.5) must balance $a p^{\gamma}$, so we choose

$$
\gamma=1-\frac{1}{\alpha}, \quad k=\left(\frac{\Gamma(1 / \alpha)}{\alpha a}\right)^{\alpha} .
$$

If on the other hand $a<0$, the second integral balances $a p^{\gamma}$ and thus

$$
\gamma=\frac{1}{\alpha}-2, \quad k=\left(\frac{-a}{\varepsilon \alpha \Gamma(2-1 / \alpha)}\right)^{\alpha} .
$$
of

(ii) $\gamma=-\frac{1}{2}$ : Now $\alpha=\frac{2}{3}$ and $k$ is determined as the unique positive root

$$
\frac{2 \varepsilon}{3} k^{2}+\frac{a}{\sqrt{\pi}} k^{3 / 2}-\frac{3}{4}=0
$$

that is

$$
k=\frac{3 a}{4 \varepsilon \sqrt{\pi}}\left(\sqrt{\left(1+\frac{2 \pi \varepsilon}{a^{2}}\right)}-1\right) .
$$

Note that as $\varepsilon \rightarrow 0, k \rightarrow 3 \sqrt{ } \pi /(4 a)$.

(iii) $-\frac{1}{2}<\gamma<0$ : No matter which integral we choose in (4.5) to balance $a p^{\gamma}$, the other integral will be more singular than $p^{\gamma}$ (see Figure 2). The only 
way to produce a term to balance $a p^{\gamma}$ is to choose $\alpha=\frac{2}{3}$ and $k=\frac{1}{2}\left(\frac{9}{\varepsilon}\right)^{1 / 3}$ (thereby causing the terms of $O\left(p^{-1 / 2}\right)$ to cancel) and to then consider higher order terms in the expansion of $s(t)$ as $t \rightarrow \infty$.

We must therefore look at asymptotic behaviours of $s(t)$ of the form

$$
s(t) \sim k t^{2 / 3}+k_{1} t^{\alpha_{1}}+o\left(t^{\alpha_{1}}\right) \quad \text { as } t \rightarrow \infty
$$

where

$$
k=\frac{1}{2}\left(\frac{9}{\varepsilon}\right)^{1 / 3} \text { and } 0<\alpha_{1}<\frac{2}{3} .
$$

The parameters $k_{1}$ and $\alpha_{1}$ are to be found in terms of $a$ and $\gamma$.

To investigate the behaviour of the integrals in (4.5), we first set $t=$ $k^{3} \tau / p^{3}$, where $k=\frac{1}{2}\left(\frac{9}{\varepsilon}\right)^{1 / 3}$; the first integral, for example, becomes

$$
\begin{aligned}
p \int_{0}^{\infty} & \exp \left(-p k t^{2 / 3}-p^{2} t-p k_{1} t^{\alpha_{1}}\right) d t \\
\quad= & \frac{k^{3}}{p^{2}} \int_{0}^{\infty} e^{-\eta\left(\tau^{2 / 3}+\tau\right)} \exp \left(-k_{1} k^{3 \alpha_{1}} p^{1-3 \alpha_{1}} \tau^{\alpha_{1}}\right) d \tau
\end{aligned}
$$

where $\eta=k^{3} p^{-1}$. Provided $0<\alpha_{1}<\frac{2}{3}$, (which is just the condition that $t^{\alpha_{1}}=o\left(t^{2 / 3}\right)$ as $\left.t \rightarrow \infty\right)$, the term $e^{-\eta \tau^{2 / 3}}$ controls the asymptotic behaviour as $p \rightarrow 0$. In this case a straightforward application of Laplace's method [4] gives the estimate

$$
\begin{aligned}
p \int_{0}^{\infty} & \exp \left(-p k t^{2 / 3}-p^{2} t-p k_{1} t^{\alpha_{1}}\right) d t \\
& \sim \frac{3 \sqrt{\pi}}{2 k^{3 / 2}} p^{-1 / 2}-\frac{3 k_{1}}{2 k^{\left(3+3 \alpha_{1}\right) / 2}} \Gamma\left(\frac{3}{2}\left(1+\alpha_{1}\right)\right) p^{\left(1 / 2-3 \alpha_{1} / 2\right)} \frac{-6}{k^{2}}+o(1) .
\end{aligned}
$$

A similar calculation can be made for the second integral in (4.5); in this case, however, some care must be taken in dealing with the lower limit of integration, as the integrand will not be integrable at $t=0$ if $\alpha_{1} \in(0,1 / 3]$. As we are concerned with the asymptotic behaviour of $s(t)$ as $t \rightarrow \infty$, however, the lower limit can be replaced by any finite nonzero constant if necessary.

After a lengthy calculation, we find the following estimate:

$$
\begin{aligned}
p \int_{0}^{\infty} & e^{-p s-p^{2} t} d t-\varepsilon \int_{0}^{\infty} \dot{s}^{2} e^{-p s-p^{2} t} d t \\
\sim & -\varepsilon k_{1} k^{-3\left(\alpha_{1}-1\right) / 2} \Gamma\left(\left(3 \alpha_{1}-1\right) / 2\right) \alpha_{1}\left(3 \alpha_{1}+1\right) p^{-\left(3 \alpha_{1}-1\right) / 2} \\
& +2 \varepsilon \alpha_{1} k_{1}^{2} k^{\left(6 \alpha_{1}-3\right) / 2} H(p)-3 \varepsilon \alpha_{1}^{2} k_{1}^{2} k^{-\left(6 \alpha_{1}-3\right) / 2} F(p) / 2 \\
& +o\left(p^{-\left(6 \alpha_{1}-3\right) / 2}\right) \text { as } p \rightarrow 0
\end{aligned}
$$




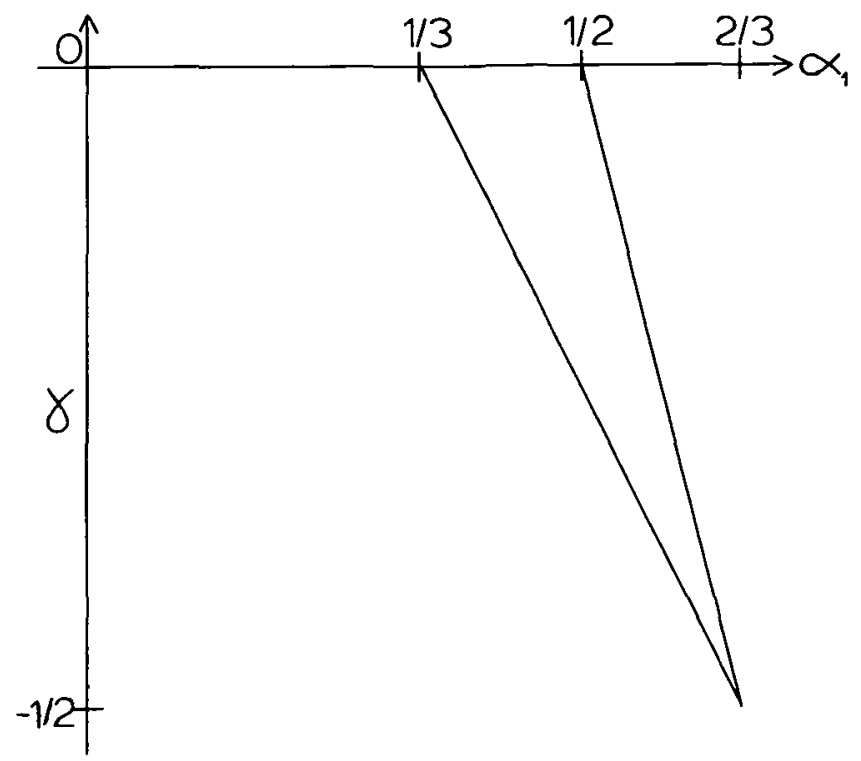

FIgURE 3. Relation between $\gamma$ and $\alpha_{1}$ for $\delta=0, \varepsilon>0$.

where

$$
\begin{gathered}
H(p)= \begin{cases}\Gamma\left(\frac{6 \alpha_{1}-1}{2}\right) p^{\left(6 \alpha_{1}-3\right) / 2}, & \frac{1}{6}<\alpha_{1}<\frac{2}{3} \\
O(p), & 0<\alpha_{1} \leq \frac{1}{6}\end{cases} \\
F(p)= \begin{cases}\Gamma\left(\frac{6 \alpha_{1}-3}{2}\right) p^{-\left(6 \alpha_{1}-3\right) / 2}, & \frac{1}{2}<\alpha_{1}<\frac{2}{3} \\
O(\log p), & 0<\alpha_{1} \leq \frac{1}{2}\end{cases}
\end{gathered}
$$

As previously, this allows us to choose $\alpha_{1}$ in terms of $\gamma$ (Figure 3). We therefore have $s(t) \sim k t^{2 / 3}+k_{1} t^{\alpha_{1}}$ as $t \rightarrow \infty$ if $-\frac{1}{2}<\gamma<0$, where $k=$ $\frac{1}{2}\left(\frac{9}{\varepsilon}\right)^{1 / 3}, \alpha_{1}=(1-2 \gamma) / 3$, and where $\frac{1}{3}<\alpha_{1}<\frac{2}{3}$ and $k_{1}$ is determined by $-\varepsilon k_{1} k^{-3\left(\alpha_{1}-1\right) / 2} \alpha_{1}\left(3 \alpha_{1}+1\right) \Gamma\left(\left(3 \alpha_{1}-1\right) / 2\right)=a$.

\section{Summary for the planar problem}

We have discussed the asymptotic behaviour of a one-dimensional Stefan problem with the kinetic condition $u=-\varepsilon \dot{s}(t)$ at the free boundary, and initial data $\varphi(x) \rightarrow-1-\delta$ as $x \rightarrow \infty$. We have investigated the cases $\varepsilon$ nonnegative, $\delta$ negative, zero and positive respectively. To summarise, we display our results in Figure 4.

(I) $\varepsilon=0, \delta>0$ : finite time blow-up. 


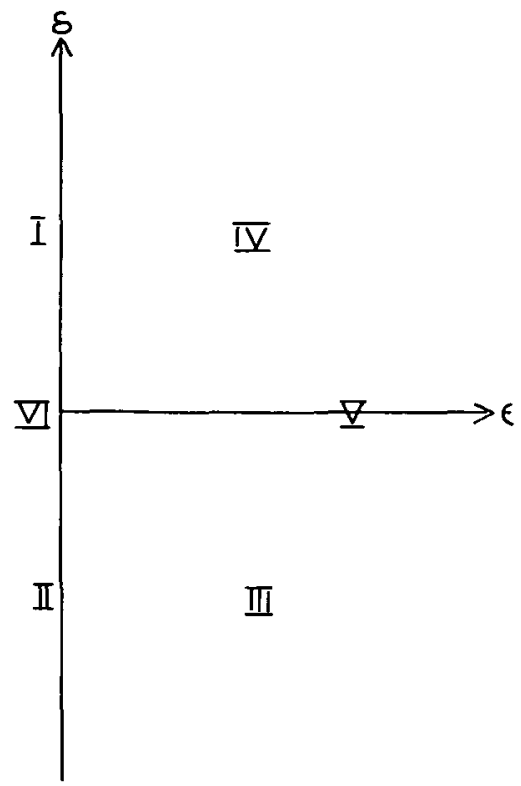

Figure 4. Regions of existence of classes of solution in the $\varepsilon-\delta$ plane.

(II) $\varepsilon=0, \delta<0$ : similarity solutions exist with $s(t)=\beta \sqrt{t}$ and $\varphi(x) \equiv$ $-1-\delta$. For other $\varphi(x)$ with $\varphi(\infty)=-1-\delta, s(t) \sim \beta \sqrt{t}$ as $t \rightarrow \infty$ provided that no blow-up occurs.

(III) $\varepsilon>0, \delta<0$ : similarity solutions (but no travelling wave solution) exist with $s(t)=\beta \sqrt{t}$; for other initial data the free boundary is asymptotic to $\beta \sqrt{t}$ as $t \rightarrow \infty$.

(IV) $\varepsilon>0, \delta>0$ : travelling wave solutions (but no similarity solution) exist with $s(t)=\delta t / \varepsilon$; for other initial data the free boundary is asymptotic to $\delta t / \varepsilon$ as $t \rightarrow \infty$.

(V) $\varepsilon>0, \delta=0: s(t) \sim k t^{\alpha}$ with $\frac{1}{2}<\alpha<1$, as $t \rightarrow \infty$. Furthermore, if $\hat{\varphi}(p)$, the Laplace transform of $\varphi(x)$, has the form

$$
\hat{\varphi}(p)=-p^{-1}+a p^{\gamma}+o\left(p^{\gamma}\right) \text { as } p \rightarrow 0
$$

(i) If $-1<\gamma<-\frac{1}{2}, a>0$, then $\alpha=1 /(1-\gamma), k=(\Gamma(1 / \alpha) / \alpha a)^{\alpha}$.

(ii) If $-1<\gamma<-\frac{1}{2}, a<0$, then $\alpha=1 /(2+\gamma)$, $k=(-a / \varepsilon \alpha \Gamma(2-1 / \alpha))^{\alpha}$.

(iii) If $\gamma=-\frac{1}{2}$, then $\alpha=\frac{2}{3}, k=(3 / 4 \varepsilon \sqrt{\pi})\left[\sqrt{a^{2}+2 \pi \varepsilon}-a\right]$.

(iv) If $-\frac{1}{2}<\gamma<0, s(t) \sim k t^{2 / 3}+k_{1} t^{\alpha_{1}}$, as $t \rightarrow \infty$, where

$\left.k=\frac{1}{2}\left(\frac{9}{\varepsilon}\right)^{1 / 3}, \alpha_{1}=\frac{1-2 \gamma}{3}, k_{1}=-a k^{\left(3 \alpha_{1}-3\right) / 2} / \varepsilon \alpha_{1}\left(1+3 \alpha_{1}\right) \Gamma\left(3 \alpha_{1}-1\right) / 2\right)$. 
(VI) $\varepsilon=\delta=0$ :

(i) If $\varphi(x)=-1+\varphi_{0}(x)$ with $\hat{\varphi}_{0}(p)$ finite and nonzero at $p=0$ then $s(t) \sim V t$ as $t \rightarrow \infty$, where $V=1 / \hat{\varphi}_{1}(0)$. This includes the travelling wave solution (2.5) as a special case.

(ii) If $\hat{\varphi}(p)$ has the same form as $(5.1),-1<\gamma<0$, then $s(t) \sim$ $k t^{\alpha}$ with $\frac{1}{2}<\alpha<1$, as $t \rightarrow \infty$, where $\alpha=1 /(1-\gamma), k=$ $(\Gamma(1 / \alpha) / \alpha a)^{\alpha}$.

(iii) No solution exists for unit step function initial data.

(iv) If $\hat{\varphi}(p)$ has the form (5.1) for $0<\gamma<1$ then $s(t) \sim k t^{\alpha}$ with $k$ and $\alpha>1$ determined as in (ii). In this case $\dot{s}(t)$ is unbounded as $t \rightarrow \infty$ and we have "infinite-time" blow-up.

\section{Three-dimensional solutions with radial symmetry}

We briefly describe the extension of our previous results to a radially symmetric three-dimensional problem with an extra term incorporating surface tension effects at the free surface via a Gibbs-Thomson condition. The spherical version of the problem (1.1)-(1.4) is

$$
\begin{array}{cc}
u_{t}=r^{-2}\left(r^{2} u_{r}\right)_{r}, & s(t)<r<\infty \\
u=-\varepsilon \dot{s}-2 \sigma / s, & r=s(t) \\
u_{r}=-\dot{s}, & r=s(t) \\
u(r, 0)=\varphi(r), & s(0) \leq r<\infty,
\end{array}
$$

where $\sigma \geq 0$ is the dimensionless surface tension. If we introduce a new variable

$$
v(r, t)=r u(r, t)
$$

then $v(r, t)$ satisfies

$$
\begin{gathered}
v_{t}=v_{r r}, \quad s(t)<r<\infty \\
v=-\varepsilon s \dot{s}-2 \sigma, \quad r=s(t) \\
v_{r}=-(\varepsilon+s) \dot{s}-2 \sigma / s, \quad r=s(t)
\end{gathered}
$$

with initial data

$$
v(r, 0)=r \varphi(r)=\psi(r),
$$

say, where $\varphi(r)$ has the same form as (1.5).

We first mention that the problem (6.1)-(6.4) can blow up in finite time if $\varepsilon=0$ and $\delta>0$ (even with surface tension), and that when $\delta<0$ there is 
a similarity solution with $s(t)=\beta \sqrt{t}$, where $\beta$ is to be determined from

$$
\frac{\beta^{2}}{2}\left(1-\beta e^{\beta^{2} / 4} \int_{\beta / 2}^{\infty} e^{-x^{2}} d x\right)=1+\delta
$$

(for details, see [2] and references therein). This similarity solution includes both surface tension and kinetic undercooling.

When $\delta>0$ there is a pseudo-travelling-wave solution ${ }^{3}$

$$
\begin{aligned}
v(r, t)= & -(1+\varepsilon V) r+2(1 / V-\sigma)+[2(V t-1 / V)-r] e^{-V(r-V t)} \\
& -\frac{2 \sigma}{V \sqrt{t}} e^{-r^{2} / 4 t} \int_{-r / 2 \sqrt{ } t}^{(r-2 V t) / 2 \sqrt{t}} e^{y^{2}} d y, \\
& s(t)=V t,
\end{aligned}
$$

where $V=\delta / \varepsilon$. For $\varepsilon=\delta=0$, this is also a solution, for arbitrary $V>0$. It is singular with $v=O\left(1 / r^{2}\right)$ at the origin as $t \rightarrow 0+$, but this can be overcome by changing the time origin.

We now investigate the large-time behaviour of the free boundary $s(t)$ for problem (6.1)-(6.4). As previously, we define the Laplace transform by (4.1). This reduces (6.1)-(6.4) to an integral equation formulation. By a straightforward calculation, we get the integral equation

$$
\begin{aligned}
\psi(p)= & -d \hat{\varphi} / d p=-e^{-p s(0)}\left[p^{-2}+(2 \sigma+2 \varepsilon+(1+\varepsilon p) s(0)) p^{-1}\right] \\
& +\int_{0}^{\infty}\left[1+2 \varepsilon p+(1+\varepsilon p) p s(t)-\frac{2 \sigma}{s(t)}-\varepsilon s(t) \dot{s}(t)^{2}\right] e^{-p s-p^{2} t} d t
\end{aligned}
$$

Repeating the method used in Section 4 we can find similar asymptotic results. Our results here confirm the numerical solutions of Schaefer and Glicksman [13].

For brevity, we state the main results only.

(1) $\varepsilon>0, \delta>0$ : the asymptotic behaviour of $s(t)$ is $V t$ and $V=\delta / \varepsilon$.

(2) $\varepsilon>0, \delta<0$ : the asymptotic behaviour of $s(t)$ is $\beta \sqrt{t}$ and $\beta$ is determined by (6.5). Note that this is independent of both $\varepsilon$ and $\sigma$.

(3) $\varepsilon>0, \delta=0$ : the asymptotic behaviour of $s(t)$ is $k t^{\alpha}$ with $\frac{1}{2}<\alpha<1$. In particular, if

$$
\hat{\psi}(p)=-e^{-p s(0)} p^{-2}+a p^{\gamma-1}+o\left(p^{\gamma-1}\right), \quad \text { as } p \rightarrow 0
$$

where $-1<\gamma<0$, then

(a) if $-1<\gamma<-\frac{1}{2}, a>0$, then $\alpha=1 /(1-\gamma), k=\left((1+\alpha) \Gamma(1 / \alpha) / \alpha^{2} a\right)^{\alpha}$;

(b) if $-1<\gamma<-\frac{1}{2}, a<0$, then $\alpha=1 /(2+\gamma), k=(-a / \varepsilon \alpha \Gamma(3-1 / \alpha))^{\alpha}$;

\footnotetext{
${ }^{3}$ This solution does not appear to have been noted previously.
} 
(c) if $\gamma=-\frac{1}{2}$ then $\alpha=\frac{2}{3}, k$ is to be determined from

$$
\frac{\varepsilon}{3} k^{3}+\frac{a}{\sqrt{\pi}} k^{3 / 2}-\frac{15}{8}=0
$$

(d) if $-\frac{1}{2}<\gamma<0$, then $\alpha=\frac{2}{3}, k=\frac{3}{2}\left(\frac{5}{3 \varepsilon}\right)^{1 / 3}$, and we proceed to higher order terms as above.

(4) $\varepsilon=\delta=0$.

(a) If $\hat{\psi}(p)$ has the form (6.7) and $-1<\gamma<0$ then

$$
\alpha=\frac{1}{1-\gamma}, \quad k=\left[\frac{1+\alpha}{a \alpha^{2}} \Gamma\left(\frac{1}{\alpha}\right)\right]^{\alpha},
$$

provided $k$ is real and positive.

(b) If $\hat{\psi}(p)$ has the form (6.7) with $\gamma=0$ then $s(t) \sim 2 t /(a+2 \sigma)$ as $t \rightarrow \infty$, provided $a+2 \sigma>0$.

(c) If $\hat{\psi}(p)$ has the form (6.7) for $\gamma>0$ and $\sigma>0$ then $s(t) \sim t / \sigma+$ $o(t)$ as $t \rightarrow \infty$. The precise form of the $o(t)$ term is determined by the higher order terms in $\hat{\psi}(p)$.

(d) If $\hat{\psi}(p)$ has the form (6.7) for $0<\gamma<1$ and $\sigma=0$ then $s(t) \sim k t^{\alpha}$, where

and we have "infinite-time" blow-up.

$$
\alpha=\frac{1}{1-\gamma}, \quad k=\left[\frac{1+\alpha}{a \alpha^{2}} \Gamma\left(\frac{1}{\alpha}\right)\right]^{\alpha}
$$

\section{References}

[1] H. S. Carslaw and J. C. Jaeger, Conduction of heat in solids (Oxford University Press, 1959).

[2] J. Chadam, S. D. Howison and P. Ortoleva, "Existence and stability for spherical crystals growing in a supersaturated solution", IMA J. Appl. Math. 39 (1987) 1-15.

[3] J. Chadam and P. Ortoleva, "The stability effect of surface tension on the development of the free boundary in a planar, one-dimensional, Cauchy-Stefan problem", IMA J. Appl. Math. 30 (1983) 57-66.

[4] E. T. Copson, Asymptotic expansions (Cambridge University Press, 1965).

[5] S. R. Coriell and R. F. Sekerka, "Oscillatory morphological instabilities due to nonequilibrium segregation', J. Crystal Growth 61 (1983) 499-508.

[6] A. B. Crowley, "Some remarks on non-equilibrium solidification problems", in Free and moving boundary problems, (eds. K. H. Hoffman and J. Sprekels), (Pitman, 1989).

[7] A. Fasano and M. Primicerio, "New results on some classical parabolic free boundary problems", Quart. Appl. Math. 38 (1981) 439-460.

[8] G. A. Garinberg and O. M. Chekmareva, "Motion of phase interface in Stefan problems", Sov. Phys. Tech. Phys. 15 (1971) 1579.

[9] S. D. Howison, J. R. Ockendon and A. A. Lacey, "Singularity development in moving boundary problems", Quart. J. Mech. Appl. Math. 38 (1985) 343-360.

[10] S. Krukowski and L. A. Turski, "Time-dependent solution for a spherically symmetric freezing precipitate", J. Crystal Growth 58 (1982) 631-635. 
[11] A. A. Lacey and J. R. Ockendon, "Ill-posed boundary problems", Control Cybernet. 14 (1985) 275-296.

[12] G. Lamé and B. P. Clapeyron, "Memoire sur la solidification par refroidissement d'un globe liquide", Annales Chimie Physique 47 (1831) 250-256.

[13] R. J. Schaefer and M. E. Glicksman, "Fully time-dependent theory for the growth of spherical crystal nuclei", J. Crystal Growth 5 (1969) 44-58.

[14] A. Visintin, "Stefan problem with a kinetic condition at the free boundary", Ann. Math. Pura Appl. (to appear).

[15] W. Xie, The Stefan problem with a kinetic condition at the free boundary, Preprint 1988. 